\title{
Classifying Wheelchair Propulsion Patterns with a Wrist Mounted Accelerometer
}

\author{
Brian French \\ ECE Department \\ Carnegie Mellon University \\ Pittsburgh PA 15213 \\ +14122683372 \\ bfrench@ece.cmu.edu
}

\author{
Asim Smailagic \\ ICES \\ Carnegie Mellon University \\ Pittsburgh PA 15213 \\ $+14122687863$ \\ asim@cs.cmu.edu
}

\author{
Dan Siewiorek \\ $\mathrm{HCl}$ Institute \\ Carnegie Mellon University \\ Pittsburgh PA 15213 \\ $+14122682570$ \\ dps@cs.cmu.edu
}

\author{
Vishnu Ambur \\ Department of Biomedical Eng. \\ Georgia Institute of Technology \\ Atlanta, Georgia 30322 \\ $+14047279874$ \\ gtg785a@gmail.com
}

\author{
Divya Tyamagundlu \\ ECE Department \\ Carnegie Mellon University \\ Pittsburgh PA 15213 \\ +14125967405 \\ divyatyam@cmu.edu
}

\begin{abstract}
In this paper, we describe a manual wheelchair propulsion classification system which recognizes different patterns using a wrist mounted accelerometer. Four distinct propulsion patterns have been identified in a limited user study. This study is the first attempt at classifying wheelchair propulsion patterns using lowfidelity, body-worn sensors. Data was collected using all four propulsion patterns on a variety of surface types. The results of two machine learning algorithms are compared. Accuracies of over $90 \%$ were achievable even with a simple classifier such as kNearest Neighbor $(\mathrm{kNN})$. Being able to identify current propulsion patterns and provide real-time feedback to novice and expert wheelchair users is potentially useful in preventing future repetitive use injuries.
\end{abstract}

\section{Keywords}

eWatch, wearable sensors, machine learning, manual wheelchair, propulsion patterns

\section{INTRODUCTION}

Persons with Spinal Cord Injury or Dysfunction (SCI/D) not only experience general functional declines that are associated with the aging process but also an increase in the prevalence of overuserelated musculoskeletal injuries. There are well-documented reports of pain, due to the long-term reliance on the upper limbs for performing daily activities. Any loss of upper limb function

Permission to make digital or hard copies of all or part of this work for personal or classroom use is granted without fee provided that copies are not made or distributed for profit or commercial advantage and that copies bear this notice and the full citation on the first page. To copy otherwise, to republish, to post on servers or to redistribute to lists, requires prior specific permission and/or a fee.

BodyNets 2008, March 13-15 Tempe, Arizona, USA

Copyright @ 2008 ICST 978-963-9799-17-2

DOI 10.4108/ICST.BODYNETS2008.2963 significantly affects mobility and independence [5].

The data indicate that upper limb pain is very common in manual wheelchair users, with carpal tunnel syndrome present in between $49 \%$ and $73 \%$ of individuals $[6,7]$. Recognizing the significance of upper limb pain and injury and the negative impact on quality of life, a recently published monograph provides concise ergonomic and equipment recommendations based on review of published evidence based research [8]. The guidelines address reducing the frequency of repetitive upper limb tasks, minimizing forces required to complete tasks and minimizing extremes of wrist and shoulder motions. Also within the guidelines are specific recommendations related to wheelchair propulsion technique, wheelchair type and configuration, and transfer activities.

There have been limited 'real-world' studies on persons with SCI who are non-ambulatory and use wheelchairs for their primary means of mobility. In order to understand the etiology of upper limb injuries and pain, it is necessary to monitor the actual usage of the upper limbs of persons with SCI.

The goal of this project is to develop a portable monitoring device along with context-aware software capable of quantifying and recognizing wheelchair propulsion patterns. Such a device will allow clinicians to collect real-world data on their patients' daily propulsion patterns. The classification task described in the remainder of this paper is a first step towards building a Virtual Coach for training wheelchair users in proper propulsion technique.

\section{FOUR PROPULSION PATTERNS}

Based on previous work on wheelchair propulsion biomechanics [1], a good wheelchair propulsion pattern should minimize cadence (stroke frequency) and maximize stroke angle (the angle 

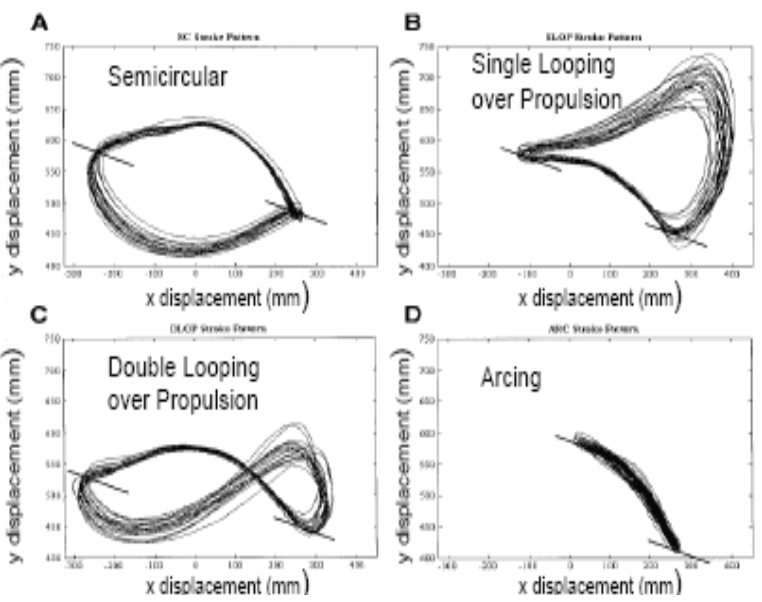

Figure 1: Four Classic Propulsion Patterns (A) Semi-circular (SC); (B) SLOP; (C) DLOP; (D) ARC; The dark bars to the right of each pattern represent the beginning of the propulsive stroke. The dark bars to the left of each pattern represent the end of the propulsive stroke and the beginning of recovery.

along the arc of the push rim from the start of propulsion to the end of propulsion). A good propulsion pattern has long and less frequent strokes when compared to a bad pattern, which has short and more frequent strokes.

Figure 1 identifies four classic propulsion patterns that wheelchair users tend to follow - semicircular, single loop over, double loop over and arcing. Of these, the recommended propulsion pattern is semi-circular, because the strokes have lower cadence and higher stroke angle as can be seen in the figure.

\section{IDENTIFYING PROPULSION PATTERNS}

Prior to this study, the only attempts at classifying wheelchair propulsion patterns were done using high-speed video motion capture technologies in the laboratory. In prior work, we have been able to classify a wide variety of human activities using lowfidelity, body-worn sensors, specifically accelerometers, and we wanted to explore using similar techniques to identify propulsion patterns.

As with our previous classification experiments, we used the eWatch - a wearable sensor and notification platform developed for context aware computing research. It has the form-factor of a wrist watch making it instantly viewable and socially acceptable, see Figure 2.

\subsection{The eWatch}

The eWatch consists of a CPU, sensors, power control, notification mechanisms, and wireless communication. The eWatch is capable of sensing temperature, light, two axes of acceleration and audio at user controllable sampling rate up to 100KHz. A MAX4061 amplifier is used for audio conditioning. We use a MEMS accelerometer to measure the planar acceleration of the user's arm. The user can be notified using a 128x64 pixel display, LEDs, a vibration motor and tone generating buzzer. Three push buttons are distributed around the outside of the

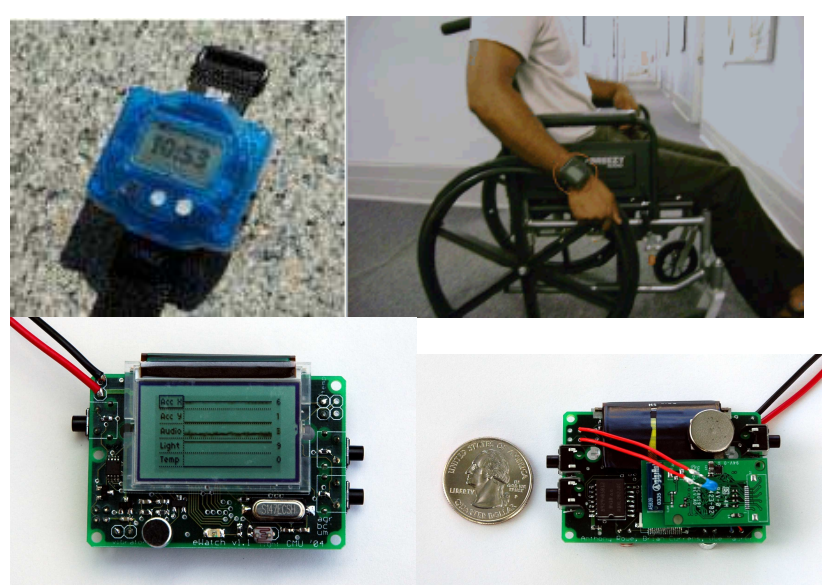

Figure 2 (top left) The eWatch developed by CMU HCII (top right) The eWatch worn on the wrist by a self-propelling wheelchair user (bottom left) the front of the eWatch circuit board with screen displaying real-time sensor data (microphone and light sensor visible at bottom of board) (bottom right) The back of the eWatch sensor board (2-d accelerometer, vibration motor and Bluetooth module are visible)

housing in the standard configuration of a digital watch. Up to 3 MB of acceleration data can be stored in onboard Flash.

\subsection{Data collection on the eWatch}

The eWatch was used to collect propulsion data from a single subject over several weeks. The subject received training on how to perform each of the four propulsion patterns from colleagues at the University of Pittsburgh's Human Engineering Research Lab (HERL). Data was collected for each pattern on five different surface types to test what impact, if any, surface resistance had on classification accuracy. The surfaces included: asphalt, tile, medium pile and low pile carpet as shown in Figure 3 and a dynamometer in the laboratory set at high resistance. A constant velocity was used throughout the study.

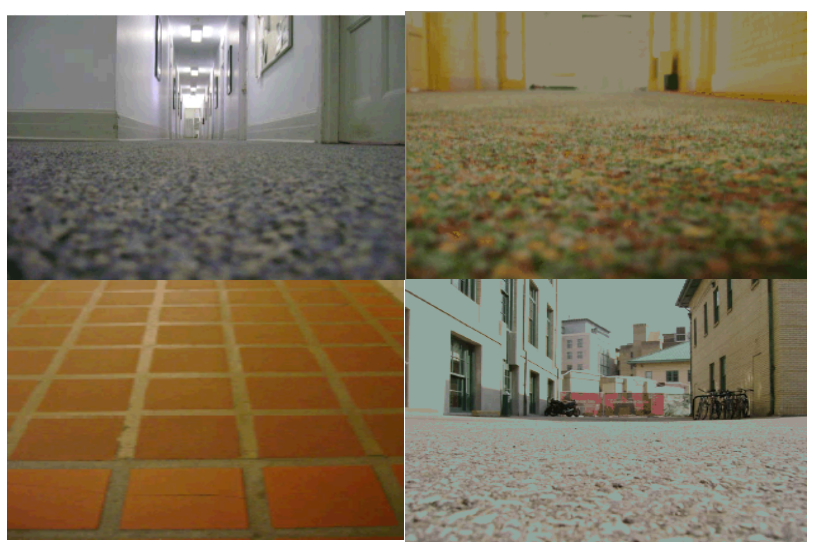

Figure 3: Trial surfaces (clockwise from top left) Low Pile Carpet; Medium Pile Carpet; Asphalt; Tile. 
The eWatch was strapped to the subject's right wrist and its twoaxis accelerometer sampled at $20 \mathrm{~Hz}$. Each trial consisted of the subject propelling over a straight 25 yards in 30 seconds. Multiple trials were performed for each propulsion/surface pair. Additional trials were performed with the eWatch worn on the subject's left wrist as well as the upper arm for some surfaces.

\section{CLASSIFICATION OF PATTERNS}

We explored classification of propulsion patterns using two common machine learning algorithms, k-Nearest Neighbor (kNN) and Support Vector Machines (SVM) with a Radial Basis Function (RBF) kernel. For a complete description of these and other machine learning algorithms see [2]. These algorithms were chosen because they have been shown to perform well on classification of accelerometer data [3,4]. We used the SVM implementation provided in the SVMLight package.

The raw accelerometer data was divided into non-overlapping windows. Using cross validation, we determined an optimal window length of approximately three seconds. This window size is long enough to ensure at least one complete cycle for all of the propulsion patterns. For each window, a set of features, both timedomain and frequency-domain, were calculated. The features included: mean, standard deviation, root mean square, median absolute deviation, zero crossing rate, mean crossing rate, range, energy and entropy. Each feature was calculated over the $\mathrm{X}, \mathrm{Y}$ and $\mathrm{X}^{2}+\mathrm{Y}^{2}$ directions, leading to a total of 27 features per data point. The data set was partitioned into training and testing data in the approximate ratio of 10:6. Cross validation on the training set was used to pick the number of neighbors for KNN and the bandwidth for SVM.

\section{RESULTS}

Figures 3 and 4 show the classification accuracy that was achieved for each surface/propulsion pattern pair using a $\mathrm{kNN}$ and SVM classifier respectively. It can be seen that the classification accuracy tends to be higher on surfaces with higher resistance (dynamometer, low carpet, asphalt), when compared to surfaces with low resistance (tile). This can be explained by the fact that low resistance surfaces allow for freer range of arm motion leading making individual patterns more difficult to recognize. The anomaly of medium-pile carpet's poor performance is due to an insufficient number of trials collected on that surface type.

It can also be seen that the classification accuracy for arcing was considerably lower than the other propulsion patterns. This can be explained by noting that arcing is a subset of each of the other patterns, and hence, is most susceptible to misclassification. By this same reasoning, DLOP is the easiest to classify because it is considerably different from the other three patterns. It is also interesting to note that arcing was the hardest pattern for human observers to recognize during the project that initially identified these patterns.

The confusion matrices in Table 1 and 2, illustrate misclassifications among the four propulsion patterns over the best (dynamometer) and worst (tile) performing surface types for the kNN classifier. The columns are true labels and the rows are classified labels. Each entry is the total number of samples predicted to have the row pattern label, when the sample was

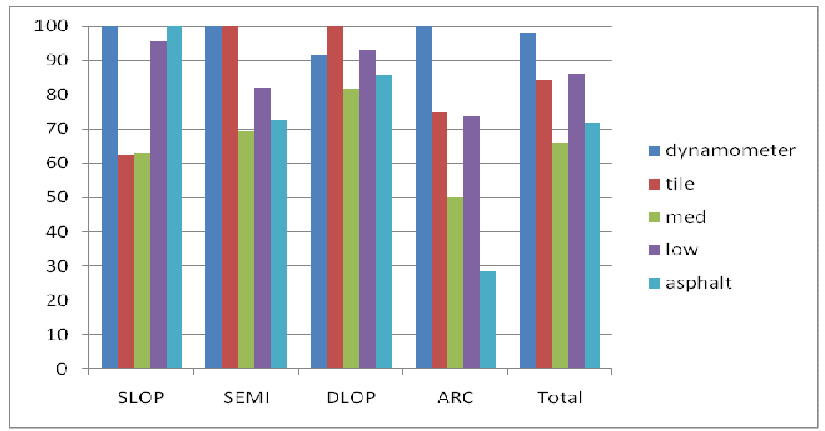

Figure 3 Classification accuracy using KNN algorithm

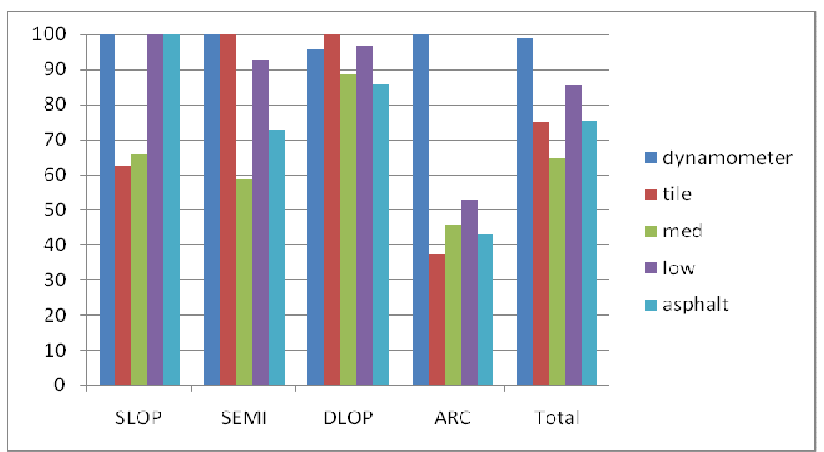

Figure 4 Classification Accuracy using SVM Algorithm

annotated with the column pattern label. Little practical improvement can be expected looking at the results for the dynamometer. However, Table 2 shows that for the tile surface, for three of the four propulsion patterns almost all misclassifications were as a single other pattern type. Therefore, finding only a few new features that improve the discrimination between the misclassified pairs of patterns should improve results significantly.

Table 1: Confusion Matrix for KNN algorithm for the dynamometer surface. Each entry is the total number of samples predicted to have the row pattern label, when the sample was annotated with the column pattern label

\begin{tabular}{|c|c|c|c|c|c|}
\hline & \multicolumn{4}{|c|}{ True Labels } \\
\hline & & SLOP & SEMI & DLOP & ARC \\
\hline \multirow{4}{*}{$\begin{array}{l}0 \\
0 \\
0 \\
0 \\
0 \\
0\end{array}$} & SLOP & 25 & 0 & 1 & 1 \\
\hline & SEMI & 0 & 35 & 0 & 0 \\
\hline & DLOP & 0 & 0 & 47 & 1 \\
\hline & ARC & 1 & 0 & 0 & 25 \\
\hline
\end{tabular}


Table 2: Confusion Matrix for KNN algorithm for the tile surface. Each entry is the total number of samples predicted to have the row pattern label, when the sample was annotated with the column pattern label

\begin{tabular}{|c|c|c|c|c|c|}
\hline & \multicolumn{4}{|c|}{ True Labels } \\
\hline & & SLOP & SEMI & DLOP & ARC \\
\hline \multirow{4}{*}{ 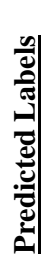 } & SLOP & 23 & 0 & 4 & 9 \\
\hline & SEMI & 0 & 32 & 0 & 0 \\
\hline & DLOP & 3 & 8 & 36 & 1 \\
\hline & ARC & 4 & 0 & 0 & 14 \\
\hline
\end{tabular}

Misclassifications are due a variety of factors. The subject tended to have slight variations in his propulsion patterns, i.e., they tend to use a combination of one or more propulsion patterns even though he intent to use only one pattern. Additionally, the 2D accelerometer values are affected by how and where the eWatch is worn by the wheelchair user. Classification can be affected by variations between the training and test datasets as a result of the position of the eWatch on the arm, the orientation of the dial, and also by whether or not the watch is worn on the dominant arm during data collection.

\section{CONCLUSION AND FUTURE WORK}

It has been demonstrated that an accurate propulsion pattern classifier can be created using only a single wrist mounted accelerometer. The higher the resistance of the surface traversed, the higher are achievable accuracies. This can be explained by the fact that lower surface resistance allows for greater freedom of arm motion during propulsion. However, evidence suggests that significant improvement in discrimination may be achievable with relatively few additional features.

The next step is to collect data from a larger group of wheelchair users. We can then refine our choices of classification algorithm and feature selections. There are also some unexplored classification variations that must be addressed. These include pattern classification between surface types, with varying velocities and most importantly across different subjects. We must also address the problem of distinguishing between propulsion and non-propulsion motion patterns.

\section{ACKNOWLEDGMENTS}

This material is based upon work supported by the National Science Foundation under Grant Nos. EEEC-540865 and 0205266, PVA Research Foundation grant, Pennsylvania Infrastructure Technology Alliance (PITA) - a collaboration among Commonwealth of Pennsylvania, Carnegie Mellon and Lehigh University, and a grant from Intel Corporation.

We would also like to thank Dr. Rosemary Cooper of the Center for Assistive Technology at University of Pittsburgh, and Dr. Ding Dan of the Health Engineering Research Lab at University of Pittsburgh for their help throughout the project.

\section{REFERENCES}

[1] Boninger ML, Souza AL, Cooper RA, Koontz AM. Propulsion Patterns and Pushrim Biomechanics in Manual Wheelchair Propulsion, Arch Phys Med Rehab, 83(5):71823, 2002

[2] Mitchell, Tom. Machine Learning. McGraw Hill 1997

[3] A. Krause, M. Ihmig, E. Rankin, D. Leong, S. Gupta, D. Siewiorek, A. Smailagic, M. Deisher, and U. Sengupta. Trading off Prediction Accuracy and Power Consumption for Context-Aware Wearable Computing. In ISWC 2005: Proceedings of the $9^{\text {th }}$ IEEE International Symposium on Wearable Computers, pages 20-26, 2005.

[4] U. Maurer, A. Smailagic, D. Siewiorek, and M. Deisher. Activity Recognition and Monitoring Using Multiple Sensors on Different Body Positions. In IEEE International Workshop on Wearable and Implantable Body Sensor Networks 2006, pp. 142-145.

[5] Sie, I.H., Waters R.L., Adkins R.H., Gellman H., Upper extremity pain in the postrehabilitation spinal cord injured patient, Arch Phys Med Rehabil., Vol. 73, 1992, pp.44-48.

[6] Burnham, R.S., Steadward, R.D., Upper extremity peripheral nerve entrapments among wheelchair athletes, Prevalence, location, and risk factors, Arch Phys Med Rehabil., Vol. 75, 1994, pp. 519-524.

[7] Aljure, J., Eltorai, I., Bradley, W.E., Lin, J.E., Johnson, B., Carpal tunnel syndrome in paraplegic patients, PARAPLEGIA, Vol. 23, 1985, pp. 182-186.

[8] Consortium for Spinal Cord Medicine: Preserving Upper Limb Function in Spinal Cord Injury: A Clinical Practice Guideline for Health-Care Professionals, Spinal Cord Medicine, Clinical Practice Guideline, 2005. 